\title{
Posición del Comité Consultivo de Inmunizaciones de la Sociedad Chilena de Infectología en relación a los cuestionamientos de las vacunas y su obligatoriedad
}

\author{
Statement from the Immunization Committee of the Chilean Infectious Diseases \\ Society in reference to vaccine refusal and mandatory policy on vaccination
}

\author{
Jan Wilhelma ${ }^{\mathrm{a}}$, Ximena Calvo ${ }^{\mathrm{b}}$, Carola Escobarc, Gabriela Moreno ${ }^{\mathrm{d}}$, \\ Liliana Veliz ${ }^{b}$, Rodolfo Villena ${ }^{e}$, Marcela Potin $^{\mathrm{f}}$ \\ aFacultad de Medicina, Clínica Alemana, Universidad del Desarrollo, Chile \\ ${ }^{b}$ Comité Consultivo de Inmunizaciones, Sociedad Chilena de Infectología, Chile \\ cHospital Dipreca Chile \\ 'Salud Ocupacional, Laboratorio Deutsche Pharma. \\ eFacultad de Medicina, Universidad de Chile, Hospital Exequiel González Cortés, Chile \\ 'División de Pediatría, Pontificia Universidad Católica de Chile
}

Artículo Publicado en Revista Chilena de Infectología 2017; 34 (6): 583-6

Fecha recibido: 7 de diciembre de 2018; Fecha aceptado: 3 de junio de 2019

\begin{abstract}
Resumen
A pesar del enorme impacto de las vacunas en la salud de la población, estas han sido y son objeto de cuestionamientos por grupos que las consideran innecesarias o inseguras y argumentan que las personas tienen el derecho a decidir sobre si estas deben ser administradas o no. Sin embargo el uso de vacunas tiene connotaciones distintas a otras decisiones en salud, ya que no vacunar impacta no solo al individuo, sino también a la comunidad que lo rodea. El inmunizar a un alto porcentaje de la población permite limitar la circulación de los agentes infecciosos, logrando la llamada inmunidad comunitaria que protege a los no vacunados por razones médicas o porque son muy pequeños. Por esta razón muchos países han definido las vacunas como obligatorias. Como Comité Consultivo de Inmunizaciones nos parece que esta estrategia es correcta sin embargo debe ser acompañada por una política de educación de la población y personal de salud sobre los beneficios y riesgos reales de las vacunas. Así mismo es necesario introducir mejoras en el sistema de notificación de reacciones adversas a vacunas haciéndolo más accesible. Adicionalmente se debe dar respuesta oportuna a los afectados por supuestas o reales reacciones a vacunas y en los casos de eventos adversos graves efectivamente asociados a vacunas. Entregar cobertura económica y acompañamiento. Finalmente es esencial la coordinación entre los diferentes actores y comunicadores para transmitir mensajes que generen confianza y respondan a las inquietudes de la población de hoy en día.
\end{abstract}

Palabras clave: Vacunas; inmunización; obligatoriedad; inmunidad comunitaria 


\begin{abstract}
Although vaccines have had a tremendous impact in public health they are questioned by certain groups that consider them unnecessary or unsafe and argue in favor of the right to decide to be vaccinated or not. However vaccines must have special considerations because unlike other medical decisions, not vaccinating has consequences not only for the individual but also for other members of the community. Immunizing a high proportion of the population limits the circulation of an infectious agent attaining what is called community or herd immunity that protects the susceptible members of the group. For this reason many countries consider vaccination mandatory as a responsibility of every citizen. This committee agrees with this view but thinks other strategies should be implemented as well, such as special educational efforts for the public and parents addressing benefits and real risks of vaccinating. Also health care professionals should be trained in vaccines. The notification system for adverse events currently available should be improved and be more accessible. Persons truly affected by adverse events due to vaccination should receive on time responses and be offered psychological and financial support. Finally all stakeholders should make coordinated efforts to work together to deliver messages that answer concerns on vaccines and bring confidence back to the public.
\end{abstract}

\section{Keywords:}

vaccines;

immunization;

mandatory;

herd immunity

\section{El problema}

Las vacunas son consideradas una de las intervenciones más costo-efectivas y equitativas en salud pública reduciendo la morbimortalidad, y mejorando la sobrevida de los individuos ${ }^{1,3}$. Su uso permite proteger a la población a lo largo de todo el ciclo vital constituyéndose en un bien público y gratuito para toda la población. En Chile las vacunas del Programa Nacional de Inmunizaciones (PNI) son obligatorias, existiendo al menos tres argumentos a favor de esto, a saber: el positivo balance entre riesgo y beneficio, el impacto en la salud de la población demostrado por sólida evidencia científica, y el principio de justicia, al disminuir las desigualdades sociales, económicas y culturales al proteger a todos por igual ${ }^{4}$. No obstante, hoy en día, la obligatoriedad es cuestionada por la sociedad, desde una lógica de derecho individual, asociado a la baja percepción de riesgo de enfermar y a la aparición de grupos con información parcial o equivoca que cuestionan los beneficios y seguridad de las vacunas.

Como cualquier intervención en salud, no existe una vacuna $100 \%$ libre de reacciones adversas, sin embargo la mayoría de ellas son leves o moderadas, y las graves son muy infrecuentes, por lo que dicho riesgo debe ser contrastado en forma justa con los beneficios. $\mathrm{Al}$ comparar por ejemplo, la frecuencia de complicaciones de la vacuna antisarampión con las de la infección por el virus salvaje parece evidente la ventaja de inmunizar ; el sarampión produce hasta un 5 a 15\% de muertes en lactantes en paises en desarrollo mientras que la vacuna, no produce muertes. Solo hay reportes aislados de complicaciones fatales en personas con graves alteraciones inmunológicas en las cuales no se debiera indicar esta vacuna por ser a virus vivo atenuado. Asi mismo la encefalitis post infección por virus sarampión se presenta con una frecuencia de 1 en 1.000 a 2.000 casos comparada con solo 1 caso por 1 millón de dosis de vacuna antisarampión ${ }^{2}$. Por ello las decisiones sobre vacunar o no hacerlo no pueden basarse solo en la presunción de evitar todo riesgo, ya que no hacer nada (y dejar de vacunar) también conlleva riesgos evidentes.

\section{Experiencia mundial con la obligatoriedad}

A pesar de los claros beneficios para la salud de la población, la aplicación práctica de la obligatoriedad varía de un país a otro. Es así como en EEUU se permiten excusas de tipo religioso o filosófico y en algunos estados hasta un $15 \%$ de los niños no reciben una o más vacunas, lo que ha conducido a brotes recientes de sarampión, y coqueluche, razón por la que varios estados de ese país han decidido reforzar la obligatoriedad ${ }^{5}$. En otras regiones como Australia el no vacunar a los niños implica pérdida de privilegios o beneficios sociales ${ }^{8}$. Por otra parte, algunos países europeos y Japón, tienen políticas más liberales de vacunación y logran altas coberturas ${ }^{6}$ pero realizan importantes inversiones en promoción y educación en el tema. El Reino Unido, no tiene políticas de obligatoriedad ${ }^{6}$, y si bien alcanza buenas coberturas globales no ha podido remontar la adherencia a la vacuna tresvírica (sarampión, parotiditis y rubeóla) luego de una publicación fraudulenta y falsa sobre la supuesta asociación de vacuna antisarampión con autismo en los niños, cuyo autor el Dr. Andrew Wakefield, hizo pública esta información y varios medios periodísticos la difundieron extensamente. A consecuencia de esto muchos padres dejaron de vacunar y las coberturas cayeron a 79,9\% con lo que muchos niños enfermaron y algunos fallecieron. Por esta razón Reino Unido está replanteando el tema de la obligatoriedad ${ }^{6,7}$. En Italia, solo se recomendaban las vacunas, pero dado que el año 2017 los casos de saram- 
pión triplicaron los del 2016, ese país decidió endurecer su legislación y aplicar penas como multas económicas o restricciones al ingreso a jardin infantil e incluso la eventual pérdida la custodia de los hijos ${ }^{9,10}$.

\section{Legislación chilena respecto a la inmunización de la población}

Las vacunas incluidas en el calendario nacional de inmunizaciones son obligatorias para los grupos poblacionales definidos por el Ministerio de Salud y ello emana de lo establecido en el artículo $32^{\circ}$ del Código Sanitario, (DFL No 725 de 1967) que establece expresamente: "El Servicio Nacional de Salud tendrá a su cargo la vacunación de los habitantes contra las enfermedades transmisibles". El mismo artículo precisa la facultad del Presidente de la República para declarar obligatoria la vacunación de la población contra las enfermedades transmisibles para las cuales existan procedimientos eficaces de inmunización. Así, el Decreto $\mathrm{N}^{\circ} 72$ de 2004, establece la delegación del Presidente de la República al Ministro de Salud en la declaración de la obligatoriedad de la vacunación. De esta forma, dicho Ministro a través del Decreto No 6/2010 y sus modificaciones, decreta la vacunación obligatoria contra las enfermedades inmunoprevenibles que corresponda. Es más, el Minsal debe garantizar el acceso a las acciones de promoción, protección y recuperación de la salud y de rehabilitación de la persona enferma; así como el de coordinar, controlar y, cuando corresponda, ejecutar tales acciones según señala el Artículo. 1 DFL 1/05 Minsal.

Si bien la Ley de Derechos y Deberes de los Pacientes No 20.584, reconoce en su artículo 14 la autonomía de la persona para rechazar un determinado tratamiento médico, indicando en su inciso primero: "Toda persona tiene derecho a otorgar o denegar su voluntad para someterse a cualquier procedimiento o tratamiento vinculado a su atención de salud, con las limitaciones establecidas en el artículo 16", en el caso de las vacunas ello está en contraposición con lo que se expone en este mismo artículo que en su inciso segundo que declara "Este derecho de elección no resulta aplicable cuando, como producto de la falta de esta intervención, procedimiento o tratamiento, se ponga en riesgo la salud pública, en los términos establecidos en el Código Sanitario. De esta circunstancia deberá dejarse constancia por el profesional tratante en la ficha clínica de la persona”.

De esta forma, es claro que la vacunación, como herramienta de bien público, se encuentra dentro de la excepción de la regla del artículo 14 que limita el derecho del paciente para otorgar o rechazar un tratamiento médico, dado que ello está enmarcado dentro del principio más general que señala que la libertad de un individuo en uso de su autonomía personal, de hacer o no hacer algo, está limitado cuando ello afecta la libertad o los derechos de otra persona, en este caso del colectivo social, pues al rechazar la vacunación se está poniendo en riesgo al resto de la población. Por lo tanto, garantizar el acceso a una determinada vacuna, no solo es una facultad del Estado de acuerdo a lo relatado en los puntos anteriores, sino que también es un deber de éste en caso de tratarse de un niño, niña o adolescente, dado que el Estado se comprometió a que ellos disfruten del más alto nivel de salud de los servicios sanitarios, en este caso, de la vacunación.

\section{Inmunidad comunitaria: medida solidaria en salud}

El porcentaje de cobertura requerido por una vacuna para alcanzar la inmunidad comunitaria es variable pero para muchas de ellas es cercano al 90-95\%, ya que por muy efectiva que sea una vacuna nunca lo es en absolutamente todos los vacunados, por condiciones ya sea asociadas a enfermedades crónicas o factores genéticos no predecibles. Por ello es esencial no permitir descensos en las coberturas aunque sea menores, pues la evidencia muestra que favorecen la aparición de brotes.

Desde el punto de vista ético, lo anterior justifica la obligatoriedad por el principio de solidaridad, que va más allá del derecho a decidir sobre la vacunación individual, destacando el bien superior de proteger a la totalidad de la población gracias a una alta cobertura.

\section{Posición del CCI}

Nuestra posición como Comité Consultivo de Inmunizaciones (CCI) de la Sociedad Chilena de Infectología es que Chile debe mantener la obligatoriedad de vacunar a los niños. Es más, invitamos a reforzarla, pero, dado que nuestra sociedad ha cambiado y la obligatoriedad es cuestionada por la ciudadanía se hacen los cambios necesarios para adecuarse a los tiempos actuales y por ello proponemos diez medidas que apuntan al fortalecimiento de la vacunación de la población:

1. Exigir que todos los niños estén con su calendario de vacunas PNI al día antes del ingreso a Salas cunas y Jardines Infantiles del país, acompañando esta exigencia de una política de educación de la población en temas de prevención de infecciones a través de las vacunas.

2. En el caso de la vacunación escolar, entrega de información oficial y por escrito a los padres y/o apoderados respecto a las vacunas, al inicio del año académico respectivo y previo a su administración, idealmente asociado a actividades educativas presenciales lideradas por profesores entrenados en el tema. 
3. Acotar la posibilidad de ser eximido temporalmente o definitivamente de la vacunación solo para condiciones médicas, demostradas que hagan sospechar una potencial reacción grave a la vacuna a traves de un certificado médico visado por la autoridad sanitaria correspondiente.

4. Difundir y hacer más accesible y simple el sistema existente de notificación obligatoria de Eventos Supuestamente Asociados a Vacunas e Inmunizaciones (ESAVI); manteniendo el seguimiento de las reacciones moderadas y graves por Farmacovigilancia del Instituto de Salud Pública (ISP) ${ }^{11}$. A su vez, reforzar la educación en Farmacovigilancia de vacunas en los médicos y equipos de salud.

5. Diseñar un procedimiento efectivo de retroalimentación, educación y acompañamiento de las personas afectadas por ESAVIS.

6. Asegurar para los casos de eventos adversos causados por vacunas una cobertura estatal oportuna de los costos derivados de su diagnóstico, manejo y rehabilitación. Esto previa calificación según evidencia médica disponible con la participación del equipo de Farmacovigilancia del ISP. En casos graves y calificados se deberá además entregar una compensación económica.

7. Disponer de programas de educación continua en vacunas para los profesionales y técnicos que trabajen en el área de inmunizaciones (TENS, Enfermeras(os) directivos de los Servicios de Salud y médicos de todo el país). A su vez, entrega de información científica a parlamentarios integrantes de la Comisión de Salud.

8. Desarrollar una adecuada coordinación de la comunicación con los medios tradicionales y redes sociales de los distintos actores que trabajan en vacunas a saber, el Comité Asesor de Vacunas e Inmunizaciones (CAVEI), Comité Consultivo de Inmunizaciones de la Sociedad Chilena de Infectología Sociedad Chilena de Pediatría, Sociedad Chilena de Epidemiología, Colegios de Enfermeras e ISP para globalizar mensajes que den confianza a la población evitando discursos públicos contradictorios.

9. Mejorar el acceso a la información del proceso de toma de decisiones sobre políticas de inmunización a nivel nacional; a través de la publicación de las actas del CAVEI, ente técnico asesor en el tema, así como a los resultados de licitaciones y decisiones de compra de la Central Nacional de Abastecimiento (CENABAST).

10. Incluir en el CAVEI a representantes, de las diferentes sociedades científicas, universidades y otros interesados en el tema.

\section{Responsabilidades éticas}

Protección de personas y animales: Los autores declaran que los procedimientos seguidos se conformaron a las normas éticas del comité de experimentación humana responsable y de acuerdo con la Asociación Médica Mundial y la Declaración de Helsinki.

Confidencialidad de los datos: Los autores declaran que han seguido los protocolos de su centro de trabajo sobre la publicación de datos de pacientes.

Derecho a la privacidad y consentimiento informado: Los autores han obtenido el consentimiento informado de los pacientes y/o sujetos referidos en el artículo. Este documento obra en poder del autor de correspondencia.

\section{Conflictos de intereses}

Rodolfo Villena declara haber trabajado como asesor científico de vacunas para Novartis hasta 2015, y como expositor en simposios de vacunas auspiciados por Pfizer, Sanofi Pasteur, GSK y MSD.

\section{Referencias}

1. Plotkin SL, Plotkin SA. Vaccines: A short history of vaccination. En: Plotkin SA, Orenstein WA, Offit PA. Vaccines. 3era ed. Philadelphia: Saunders; 1999.

2. Redd S. Measles vaccine. En: Plotkin SA, Orenstein WA, Offit PA. Vaccines. 3era ed. Philadelphia: Saunders; 1999.

3. World Health Organization (WHO), Choosing Interventions that are Cost Effective (CHOICE). Geneva: WHO, 2009. http://www.who.int/choice/en/ Consultado 27 mayo 2017.
4. Estrategia nacional de salud para el cumplimiento de los Objetivos Sanitarios de la Década 2011-2020. Inscripción No 211.726, Registro de Propiedad Intelectual. ISBN: 978-956-348-005-4.

5. Senate Bill No. 277 CHAPTER 35 An act to amend Sections 120325, 120335 , 120370, and 120375 of, to add Section 120338 to, and to repeal Section 120365 of, the Health and Safety Code, relating to public health. Approved by Governor June 30, 2015. Filed with Secretary of State June 30, 2015.

6. NHS Immunisation Statistics England,
2015-16 Published 22 September 2016 ISBN: 978-1-78386-817-9.

7. http://content.digital.nhs.uk/pubs/ immstats1516 Consultado 24 agosto 2017.

8. http://www.immunise.health.gov.au/ Consultado el 27 de mayo de 2017.

9. http://www.trovanorme.salute.gov. it/norme/dettaglioAtto?id=59548 Consultado el 24 de agosto de 2017.

10. http://vacunasaep.org/profesionales/ noticias/italia-hace-obligatorias-vacunasinfantiles Consultado 24 agosto 2017.

11. http:///www.ispch.cl/anamed_/ farmacovigilancia/vacunas. Consultado el 21 de septiembre de 2017. 